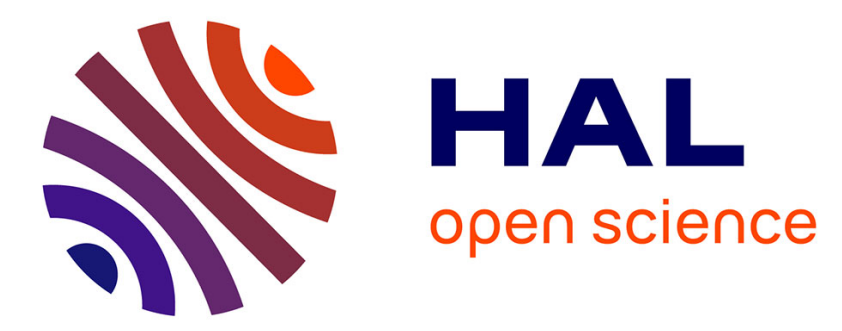

\title{
Collective water dynamics in the first solvation shell drive the NMR relaxation of aqueous quadrupolar cations
}

Antoine Carof, Mathieu Salanne, Thibault Charpentier, Benjamin Rotenberg

\section{- To cite this version:}

Antoine Carof, Mathieu Salanne, Thibault Charpentier, Benjamin Rotenberg. Collective water dynamics in the first solvation shell drive the NMR relaxation of aqueous quadrupolar cations. Journal of Chemical Physics, 2016, 145, pp.124508. 10.1063/1.4963682 . cea-01374228

HAL Id: cea-01374228

https://hal-cea.archives-ouvertes.fr/cea-01374228

Submitted on 30 Sep 2016

HAL is a multi-disciplinary open access archive for the deposit and dissemination of scientific research documents, whether they are published or not. The documents may come from teaching and research institutions in France or abroad, or from public or private research centers.
L'archive ouverte pluridisciplinaire HAL, est destinée au dépôt et à la diffusion de documents scientifiques de niveau recherche, publiés ou non, émanant des établissements d'enseignement et de recherche français ou étrangers, des laboratoires publics ou privés. 


\section{AlP $\mid \begin{aligned} & \text { The Journal of } \\ & \text { Chemical Physics }\end{aligned}$}

Collective water dynamics in the first solvation shell drive the NMR relaxation of aqueous quadrupolar cations

Antoine Carof, Mathieu Salanne, Thibault Charpentier, and Benjamin Rotenberg

Citation: The Journal of Chemical Physics 145, 124508 (2016); doi: 10.1063/1.4963682

View online: $\mathrm{http}: / / d x$. doi.org/10.1063/1.4963682

View Table of Contents: http://scitation.aip.org/content/aip/journal/jcp/145/12?ver=pdfcov

Published by the AIP Publishing

\section{Articles you may be interested in}

Role of van der Waals corrections in first principles simulations of alkali metal ions in aqueous solutions

J. Chem. Phys. 143, 194510 (2015); 10.1063/1.4935932

On the microscopic fluctuations driving the NMR relaxation of quadrupolar ions in water

J. Chem. Phys. 143, 194504 (2015); 10.1063/1.4935496

Communication: Hydration structure and polarization of heavy alkali ions: A first principles molecular dynamics study of $\mathrm{Rb}+$ and $\mathrm{Cs}+$

J. Chem. Phys. 137, 041101 (2012); 10.1063/1.4742151

Towards accurate solvation dynamics of divalent cations in water using the polarizable amoeba force field:

From energetics to structure

J. Chem. Phys. 125, 054511 (2006); 10.1063/1.2234774

Solute size effects on the solvation structure and diffusion of ions in liquid methanol under normal and cold conditions

J. Chem. Phys. 124, 084507 (2006); 10.1063/1.2172598

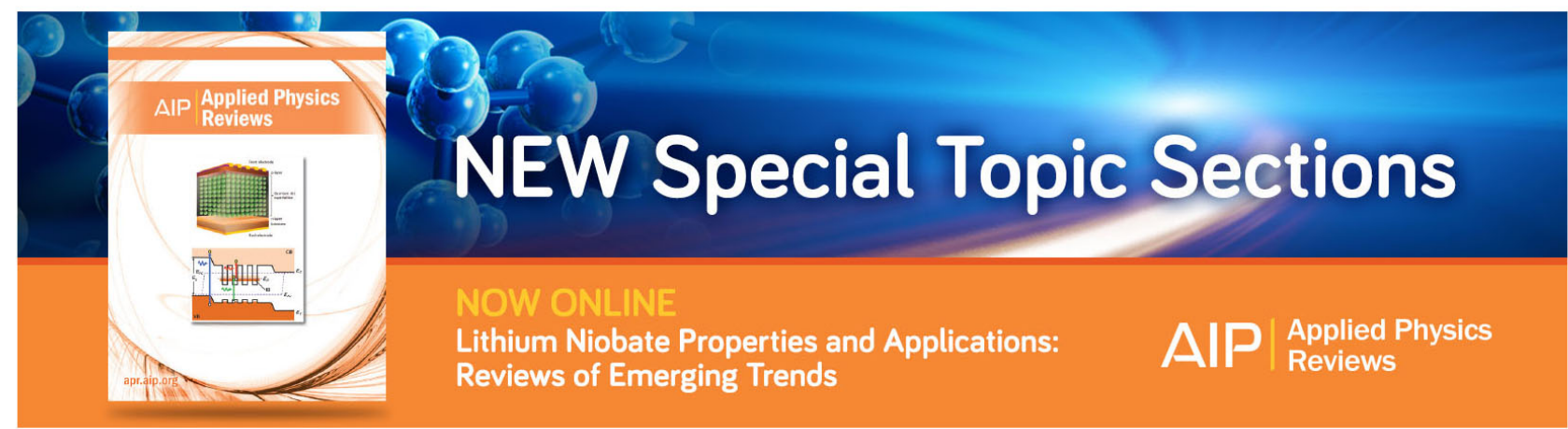




\title{
Collective water dynamics in the first solvation shell drive the NMR relaxation of aqueous quadrupolar cations
}

\author{
Antoine Carof, ${ }^{1}$ Mathieu Salanne, ${ }^{1}$ Thibault Charpentier, ${ }^{2}$ and Benjamin Rotenberg ${ }^{1}$ \\ ${ }^{1}$ Sorbonne Universités, UPMC Universités Paris 06, CNRS, Laboratoire PHENIX, Case 51, 4 place Jussieu, \\ F-75005 Paris, France \\ ${ }^{2}$ NIMBE, CEA, CNRS, Université Paris-Saclay, CEA Saclay, 91191 Gif-sur-Yvette cedex, France
}

(Received 16 May 2016; accepted 14 September 2016; published online 29 September 2016)

\begin{abstract}
Using molecular simulations, we analyze the microscopic processes driving the Nuclear Magnetic Resonance (NMR) relaxation of quadrupolar cations in water. The fluctuations of the Electric Field Gradient (EFG) experienced by alkaline and magnesium cations, which determine the NMR relaxation time, are mainly due to the dynamics of water molecules in their solvation shell. The dynamics of the ion plays a less important role, with the exception of the short-time dynamics in the lighter $\mathrm{Li}^{+}$case, for which rattling in the solvent cage results in oscillations of the EFG autocorrelation function (ACF). Several microscopic mechanisms that may a priori contribute to the decay of the EFG-ACF occur in fact over too long time scales: entrance/exit of individual water molecules into/from the solvation shell, rotation of a molecule around the ion, or reorientation of the molecule. In contrast, the fluctuations of the ion-water distance are clearly correlated to that of the EFG. Nevertheless, it is not sufficient to consider a single molecule due to the cancellations arising from the symmetry of the solvation shell. The decay of the EFG-ACF, hence NMR relaxation, is in fact governed by the collective symmetry-breaking fluctuations of water in the first solvation shell. Published by AIP Publishing. [http://dx.doi.org/10.1063/1.4963682]
\end{abstract}

\section{INTRODUCTION}

Among the plethora of experimental techniques to explore the microscopic dynamics in the condensed phase, the measurement of Nuclear Magnetic Resonance (NMR) relaxation times provides access to molecular-scale information over a broad range of time scales. NMR relaxation times characterize the return to equilibrium of an ensemble of previously excited nuclear spins, ${ }^{1,2}$ as a result of their weak coupling to a fluctuating electrostatic and magnetic environment. Extracting microscopic information from such a macroscopic measurement requires a model for the environment of the nuclei and of its dynamics. Numerical simulation has therefore proved very useful to establish such mechanisms for pure water ${ }^{3,4}$ or complex systems such as proteins ${ }^{5}$ and porous media. ${ }^{6}$ Despite their apparent simplicity and their importance in many contexts, as well as being the focus of several theoretical and simulation studies, the molecular mechanism underlying the NMR relaxation of simple aqueous ions remains elusive. There is therefore a need to address the following points: (i) What can we really learn from their NMR relaxation rates on the dynamics of water around them? (ii) How does the chemical nature of the ions change such dynamics?

In the specific case of quadrupolar mono-atomic ions in water, the coupling between the electrostatic quadrupole of the nuclei and the Electric Field Gradient (EFG) generally controls the relaxation process. While the electrostatic quadrupole moment is a nuclear property, tabulated for the different isotopes, ${ }^{7}$ the EFG is a dynamical quantity fluctuating together with the charge distribution around the considered nuclei.
In the present case, the microscopic processes driving the relaxation occur much faster $(\tau \sim 10 \mathrm{ps})$ than the inverse of the Larmor frequency $\omega_{0}=\gamma B_{0}$, with $\gamma$ the gyromagnetic ratio and $B_{0}$ the static magnetic field $(>10 \mathrm{~ns})$. In this so-called extreme narrowing regime, NMR theory relates these microscopic fluctuations to the macroscopic relaxation times,

$$
\frac{1}{T_{1}}=\frac{1}{T_{2}}=\frac{3}{8} \frac{2 I+3}{I^{2}(2 I-1)}\left(\frac{e Q}{\hbar}\right)^{2} J_{E F G}(0),
$$

where $I$ and $e Q$ are, respectively, the spin and the electrostatic quadrupole of the nuclei. $J_{E F G}(0)$ is the spectral density at zero-frequency of the EFG experienced by the nuclei, i.e., Laplace transform (at imaginary frequency $-i \omega$ ) of its time Auto-Correlation Function (ACF),

$$
J_{E F G}(\omega)=\int_{0}^{\infty}\left\langle V_{z z}(t) V_{z z}(0)\right\rangle e^{i \omega t} \mathrm{~d} t .
$$

$V_{z z}$ is the $z z$ Cartesian component of the EFG tensor in the laboratory frame and the brackets stand for an equilibrium ensemble average.

Of particular interest for simple aqueous ions is the dependence of the NMR relaxation rate on their chemical nature. One thus needs to model the effect of their mass, radius, and charge on the EFG fluctuations. This not only requires to accurately describe the $\mathrm{EFG}$ for each microscopic configuration but also to correctly capture the dynamics of the nucleus environment over the appropriate time scales. While the EFG experienced by the nucleus arises for a large part from the electronic cloud around it, this contribution 
can be captured as a linear response to the environment within the standard Sternheimer approximation, 8,9 which we recently validated using a combination of advanced quantum mechanical calculations and classical molecular dynamics. ${ }^{10}$

Early models of the EFG arising from the surrounding solvent molecules were based on analytical theories. Hertz analyzed the contribution of a single water molecule to the EFG-ACF and assumed that the cross correlations between different molecules are proportional to the latter, ${ }^{11}$ via a polarization factor calculated from continuum electrostatics. He further hypothesized that the most important molecular process is the reorientation of water molecules, captured by a Brownian model. Further analytical theories by Hynes and Wolynes $^{12}$ and later by Perng and Ladanyi ${ }^{13}$ relied instead on a continuous description of the solvent, for which an accurate description of the charge or dipole dynamics is still missing.

The pioneering works of Engström and Jönsson ${ }^{14-16}$ opened the way to the study of the NMR relaxation of aqueous cations with molecular simulation. ${ }^{10,17-21,23}$ Engström et $a l .{ }^{16}$ studied cooperative effects between water molecules and indicated that the translation of water may be more important than its reorientation. Roberts and Schnitker ${ }^{17}$ investigated cross correlations of the EFG due to different water molecules and casted doubt upon Hertz's polarization factor. Kowalewski and Odelius ${ }^{18}$ focused on the quadrupolar and dipolar relaxation mechanisms in the case of the lithium ion, also pointing out that water reorientation may play a minor role compared to translation and offering a new analysis to the self and cross terms. Quantum calculations recently offer new opportunities for the calculation of NMR relaxation rates, ${ }^{20,21}$ in order to bypass the need for a classical force field in molecular simulations. However the entailed computational cost currently does not allow to accurately sample the relevant time scales (of the order of ns).

Using molecular simulation of aqueous ions, we recently calculated NMR relaxation rates in good agreement with experimental data. ${ }^{10,22}$ This validation of the simulation model opened the way for a detailed analysis of the microscopic properties of the systems of interest. Our simulations highlighted that the fluctuations of the EFG experienced by the quadrupolar nucleus are dominated by the first hydration shell of the ions. ${ }^{23}$ We proposed a simple ansatz of the charge distribution, the Radial Dipole Model (RDM), to estimate the EFG-ACF, without further investigating the microscopic dynamics giving rise to the EFG fluctuations. This fundamental question, at the origin of all the above-cited work, remains open and the present work aims at assessing the importance of several microscopic processes.

Here we analyze the effects of the ion and water dynamics on the time-dependence of the EFG, in order to correlate the observed relaxation times with microscopic mechanisms. Specifically, we consider alkaline and magnesium cations and investigate isotopic effects, the entrance/exit from the first solvation shell and several features of water dynamics within this shell: rotation, reorientation, and vibration. This allows us to demonstrate that the decay of the EFG auto-correlation function is governed by the collective, symmetry-breaking fluctuations of water in the first solvation shell.

\section{METHODS}

\section{A. Theory}

The EFG at the nucleus site depends on the local environment of the ion, via the corresponding charge distribution, but also on the deformation of the electronic cloud of the ion itself induced by the latter. This renders the computation of the spectral density at zero frequency $J_{E F G}(0)$ difficult because it requires both the accurate evaluation of the EFG for each microscopic configuration, including in principle core electrons, and the sampling of the relevant time scales. In order to obtained statistically converged $J_{E F G}(0)$, it is necessary to sample the different environments of the ion over several nanoseconds of trajectory, despite the decay of the EFG-ACF over shorter time scales $(\tau \sim 10 \mathrm{ps})$. Such a sampling therefore remains a formidable challenge for $a b$ initio molecular dynamics. ${ }^{20}$ Fortunately, the response of the electronic cloud to the external charge distribution can be captured in the linear form of the so-called Sternheimer approximation. ${ }^{8,9}$ The NMR relaxation rates then read

$$
\frac{1}{T_{1}}=\frac{1}{T_{2}}=\frac{3}{8} \frac{2 I+3}{I^{2}(2 I-1)}\left(\frac{e Q}{\hbar}\right)^{2}\left(1+\gamma_{\infty}\right)^{2} J(0),
$$

where $J(0)$ is the spectral density at zero frequency of the "external" EFG arising from the environment of the ion and $\gamma_{\infty}$ is the nucleus-specific Sternheimer factor. ${ }^{8}$ We have recently validated this approximation in the present case of aqueous ions and computed the Sternheimer factors corresponding to the microscopic model (see Sec. II B). ${ }^{10}$ The dynamics of the EFG is then entirely governed by that of the external EFG via $J(0)$, which we compute using classical molecular dynamics simulations.

\section{B. Simulation details}

Alkaline $\left(\mathrm{Li}^{+}, \mathrm{Na}^{+}, \mathrm{K}^{+}, \mathrm{Rb}^{+}\right.$, and $\left.\mathrm{Cs}^{+}\right)$and alkaline earth $\left(\mathrm{Mg}^{2+}\right)$ cations in water at infinite dilution are modelled by a single ion and 215 water molecules in a cubic box of length $18.65 \AA$. We use a recently developed force field, based on the Polarizable Ion Model (PIM), ${ }^{24}$ in conjunction with the polarizable water model of Dang and Chang. ${ }^{25}$ The PIM is parametrized on density functional theory (DFT) calculations in order to reproduce both the $a b$ initio forces and dipoles and was shown to accurately describe the thermodynamic, structural, and dynamical properties of aqueous ions. ${ }^{24}$ As explained below, we also consider isotopic effects by considering systems where the mass of the ion is infinite (fixed ion) or where the mass of oxygen (WO model) or hydrogen (WH model) atoms is doubled.

Classical MD trajectories are generated using the $\mathrm{CP} 2 \mathrm{~K}$ simulation package. ${ }^{26}$ For each ion, 5 independent trajectories of $500 \mathrm{ps}$ in the $N V E$ ensemble are generated, obtained after annealing at $1000 \mathrm{~K}$ for $150 \mathrm{ps}$ followed by 50 ps equilibration at $298 \mathrm{~K}$, using a time step of $1 \mathrm{fs}$. The seed configurations for our annealing process are taken from a previous work on similar systems ${ }^{24}$ or built by positioning the water molecules and the ion on a regular grid. Periodic boundary conditions in all directions are used. A cutoff of $9.325 \AA$ is used for short-range interactions, while electrostatic interactions and 
the EFG are computed with Ewald summation. ${ }^{27}$ The normalized correlation function is obtained as

$$
C_{E F G}^{n o r m}(\tau)=\frac{\langle\mathbf{V}(\tau): \mathbf{V}\rangle}{\left\langle\mathbf{V}^{2}\right\rangle}=\frac{1}{\left\langle\mathbf{V}^{2}\right\rangle} \sum_{\alpha, \beta}\left\langle V_{\alpha \beta}(\tau) V_{\alpha \beta}\right\rangle
$$

where $\alpha$ and $\beta$ indicate the Cartesian components of the EFG tensor. Water molecules are treated as rigid using the SHAKE algorithm. ${ }^{28,29}$ During equilibration the temperature is maintained at the target value using a Nosé-Hoover thermostat $^{30,31}$ with a time constant of 1 ps. The EFG-ACF is computed from the EFG sampled every 5 fs along the trajectories. Error bars indicate the standard deviation among the 5 independent trajectories.

\section{RESULTS AND DISCUSSION}

The key microscopic observable for the NMR relaxation of quadrupolar nuclei, the EFG-ACF, depends on the relative positions of the nuclei and its environment and their fluctuations over time. Since the first solvation shell of the ion is the main contribution to the $\mathrm{EFG},{ }^{23}$ we examine several microscopic processes associated with their dynamics. We first analyze the effect of isotopic substitution to clarify the role of the ion dynamics, as well as water rotation and translation. We then investigate entrance/exit of water molecules from the first solvation shell. Finally, we analyze the dynamics of water in the solvation shell.

\section{A. Isotopic effects}

We first assess the role of the ion dynamics on the EFG-ACF by comparing the results obtained for each ion with that for an otherwise identical ion but with infinite mass (i.e., immobilized). This strategy, already successfully applied to obtain microscopic information on the friction coefficient of ions in water, ${ }^{32}$ allows decoupling the ion dynamics from that of the solvent. The normalized EFG-ACF, $\mathrm{C}_{\mathrm{EFG}}^{\text {norm }}$, is presented in Figure 1 for $\mathrm{Li}^{+}, \mathrm{K}^{+}, \mathrm{Cs}^{+}$, and $\mathrm{Mg}^{2+}$. As noted previously, ${ }^{23}$ the EFG-ACF decays in two steps, with a first characteristic time varying from $20 \mathrm{fs}$ for $\mathrm{Li}^{+}$to $90 \mathrm{fs}$ for $\mathrm{Cs}^{+}$and a second one of the order of $1 \mathrm{ps}$ (with a maximum for $\mathrm{K}^{+}$).

For $\mathrm{Li}^{+}$, which is lighter than water molecules, one observes oscillations with a period of $\sim 80$ fs. These oscillations disappear for the infinitely heavy isotope. They are therefore due to the rattling of the ion in its solvation shell, as suggested by the comparison between the EFG and velocity autocorrelation functions (VACF). ${ }^{23}$ The decay of the EFG-ACF for the infinitely heavy $\mathrm{Li}^{+}$proceeds in two steps, as for the other ions, with shallower and slower oscillations. During the first step of the decay, we note that the envelope of oscillations for $\mathrm{Li}^{+}$with a normal mass follows the curve for the infinitely heavy ion. This indicates that two mechanisms overlap for $\mathrm{Li}^{+}$during the first decay of the EFG-ACF: the rattling of this light ion in the solvent cage and the slower evolution of this cage over a few $100 \mathrm{fs}$.

For larger alkali ions (including $\mathrm{Na}^{+}$and $\mathrm{Rb}^{+}$, not shown), the results obtained with the normal and infinitely heavy ions are very similar. This suggests that most of the EFG
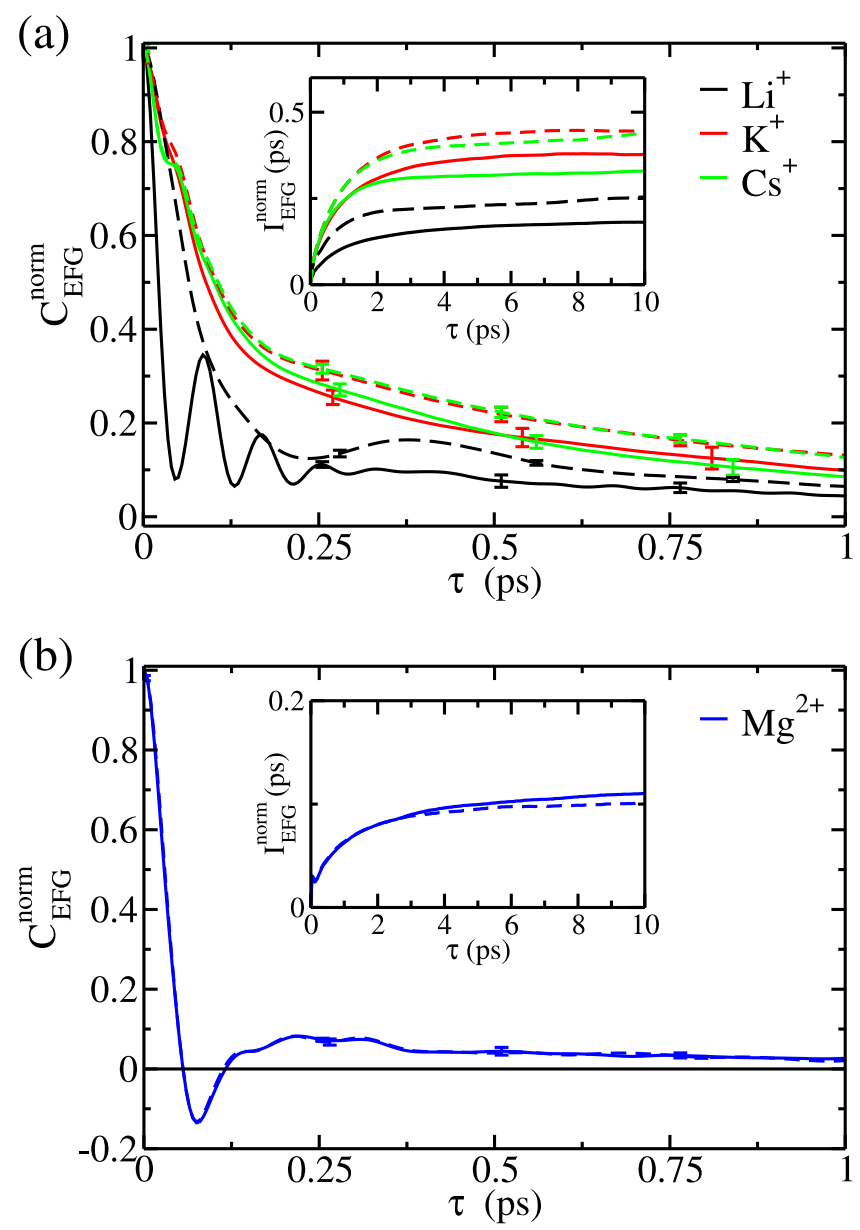

FIG. 1. Normalized electric field gradient autocorrelation functions, $C_{E F G}^{\text {norm }}$, and their integrals (inset) for aqueous ions with normal (full lines) and infinite (dashed lines) mass at infinite dilution: (a) $\mathrm{Li}^{+}, \mathrm{K}^{+}$, and $\mathrm{Cs}^{+}$and (b) $\mathrm{Mg}^{2+}$. Error bars indicate standard deviations over five independent trajectories.

fluctuations are due to the dynamics of the solvation shell. Since these ions are heavier than water molecules, their dynamics play a limited role at short times and only in the second step of the decay can we notice an effect of the ion translation. The slightly slower decay in the infinitely heavy case suggests that ion diffusion participates as a secondary mechanism to the decorrelation of the ACF. This long-term effect is best seen in the inset of Figure 1(a), which shows that the integral of $\mathrm{C}_{\mathrm{EFG}}^{\mathrm{norm}}$ converges to the plateau $(\propto J(0)$ in Eq. (3)) more slowly and to a larger value than for the ions with normal mass.

We finally note that the EFG-ACF for the heavier $\mathrm{K}^{+}$and $\mathrm{Cs}^{+}$(as well as $\mathrm{Na}^{+}$and $\mathrm{Rb}^{+}$, not shown) is almost identical. This further confirms the decoupling of the ion and water dynamics in this regime. Such a decoupling is also evident in the case of $\mathrm{Mg}^{2+}$, for which the mass has very limited influence on the EFG-ACF and its integral, as can be seen in Figure 1(b). The ACF differs markedly from that of other ions and includes a negative minimum after $\sim 80 \mathrm{fs}$. Contrary to the $\mathrm{Li}^{+}$case, this cannot be due to ion rattling in the solvent cage, since this would depend on the mass of the ion. Such a feature therefore arises from the solvent dynamics.

Table I reports the spectral densities at zero frequency $J(0)$ and $J^{\infty}(0)$ for the external EFG (see Eqs. (2) and (1)) 
TABLE I. Spectral density at zero frequency of the external electric field gradient (see Eqs. (2) and (3)) for aqueous ions with their normal mass, $\boldsymbol{J}(0)$, and for their infinitely heavy isotope, $J^{\infty}(0)$.

\begin{tabular}{lcc}
\hline \hline Ions & $J(0)\left(10^{27}\right.$ S.I. $)$ & $J^{\infty}(0)\left(10^{27}\right.$ S.I. $)$ \\
\hline $\mathrm{Li}^{+}$ & 9.37 & 12.9 \\
$\mathrm{Na}^{+}$ & 3.11 & 4.26 \\
$\mathrm{~K}^{+}$ & 2.19 & 2.62 \\
$\mathrm{Rb}^{+}$ & 1.54 & 1.85 \\
$\mathrm{Cs}^{+}$ & 0.90 & 1.20 \\
\hline $\mathrm{Mg}^{2+}$ & 1.65 & 1.74 \\
\hline \hline
\end{tabular}

obtained with the normal and infinite mass, respectively. Quantitatively, neglecting ion translation overestimates the relaxation rate by $5 \%$ for $\mathrm{Mg}^{2+}$ to $35 \%$ for $\mathrm{Li}^{+}$. The larger error in the latter case arises from the failure to capture the oscillations at short times. Summarizing the results of this section, we can conclude that the ion dynamics only plays a limited role in the EFG-ACF, with the exception of $\mathrm{Li}^{+}$, which is lighter than the water molecules. While not unexpected, such a conclusion suggests that one can focus on the water dynamics around the ion, to which we now turn.

As illustrated above, isotopic substitution allows us to assess the influence of molecular motion on the EFG-ACF. We now consider three models to analyze the role of water dynamics. The first two consist in doubling the mass of oxygen (WO model) or hydrogen (WH model) of water molecules. In a last model (WK-WO), the mass of the oxygen is doubled and the ion is fixed. Due to the large difference in mass of $\mathrm{O}$ and $\mathrm{H}$, the WO model essentially corresponds to changing the mass of the water molecule while the WH model changes mainly its moment of inertia, thus allowing to separate the contributions of translation and rotation of a single molecule to the EFG fluctuations.

The EFG-ACF for all models is reported in Figure 2. Changing the moment of inertia of the molecule (WH model)

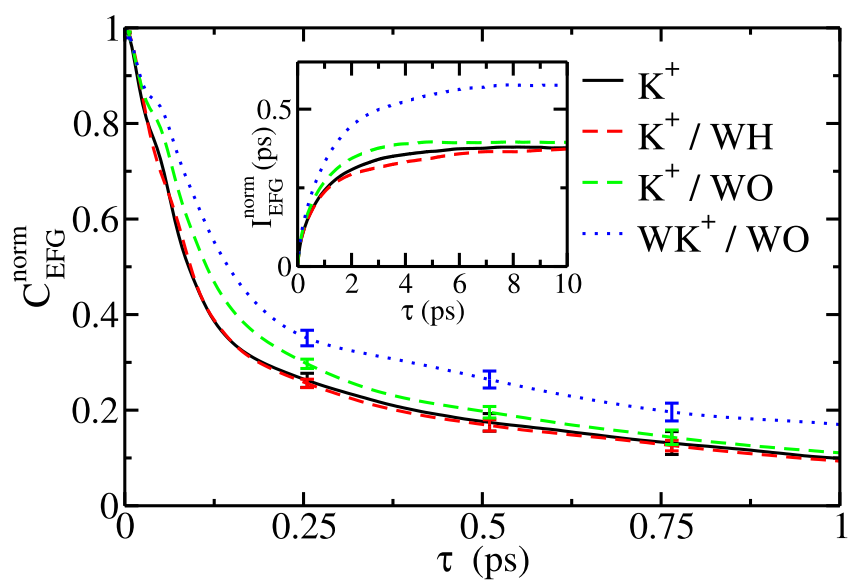

FIG. 2. Normalized correlation function $C_{E F G}^{n o r m}$ and their integrals (inset) of the EFG at the nucleus site of an aqueous potassium ion. In addition to the real system, we consider the effect of isotopic substitutions by doubling the mass of the oxygen (WO) or hydrogen (WH) of water molecules. In the last model $\left(\mathrm{WK}^{+}\right)$, the mass of the ion is infinite. Error bars indicate standard deviations over five independent trajectories.
TABLE II. Reduced mass for an ion-water pair for the standard, WK, and WO models (see text).

\begin{tabular}{lccc}
\hline \hline Model & $m_{K}\left(\mathrm{~g} \mathrm{~mol}^{-1}\right)$ & $m_{\boldsymbol{O}}\left(\mathrm{g} \mathrm{mol}^{-1}\right)$ & $m_{\text {red }}\left(\mathrm{g} \mathrm{mol}^{-1}\right)$ \\
\hline$\ldots$ & 39.1 & 16.0 & 12.3 \\
WO & 39.1 & 32.0 & 18.2 \\
WK & $\infty$ & 16.0 & 18.0 \\
WK, WO & $\infty$ & 32.0 & 34.0 \\
\hline \hline
\end{tabular}

has no effect on the decay of the EFG, showing that the reorientation of water molecules plays a limited role on the decorrelation of the EFG, hence on the NMR relaxation rate. Changing the mass of the molecule (WO model) results in a limited slowing down of the decay, comparable to that observed in Figure 1 for an infinitely heavy ion. Such a slowing down is more pronounced when the ion is infinitely heavy and the oxygen is twice heavier (WK-WO). This is consistent with the larger change in the reduced mass for an ion-water pair in this case, as can be seen in Table II. By freezing out translational or rotational water motion in the surrounding of $\mathrm{Li}^{+}$or $\mathrm{Na}^{+}$, Engström et al. ${ }^{16}$ presented preliminary evidences that the translation of the water molecules dominates the EFG fluctuations. The present analysis of isotopic substitutions confirms their results and demonstrates that the translation of water molecules is the principal cause of the EFG fluctuations for the alkali and magnesium ions.

The fact that water is lighter than most of the ions considered here explains why the dynamics of the latter plays a secondary role. The present analysis of isotopic effects suggests a simple strategy to model the dynamics of water around the ions to describe the EFG-ACF, in order to assess the effect of its charge and radius. In the first step, one could neglect the motion of the ion and focus on the dynamics of water in the external potential arising from the ion. In the second step, the dynamics of the ions could partially be taken into account by replacing the water mass by the reduced one.

\section{B. Entrance into and exit from the solvation shell}

Since the main contribution of the EFG-ACF arises from water molecules in the first solvation shell, ${ }^{23}$ one should first examine the entrance into and exit from the latter. The first solvation shell is defined as usual by introducing a cutoff distance at the first minimum of the radial distribution function (rdf). The rdf and corresponding cutoff for the present model of aqueous ions can be found in Ref. 24. Several measures have been proposed to quantify the dynamics of entrance into and exit from the solvation shell. ${ }^{33-35}$ Here we introduce for each molecule a function $S_{c}(\tau)$ equal to 1 if it remains continuously in the first solvation shell between 0 and $\tau$, and to 0 otherwise, the survival probability is given by the canonical ensemble average $\left\langle S_{c}(\tau)\right\rangle$. Other prescriptions, such as considering the presence only at times 0 and $t$ and not intermediate ones, result in similar conclusions and will not be discussed here. The survival probability is reported for $\mathrm{Li}^{+}, \mathrm{K}^{+}, \mathrm{Cs}^{+}$, and $\mathrm{Mg}^{2+}$ in Figure 3(a). For the alkaline cations, the decay is almost exponential, with characteristic times decreasing along 

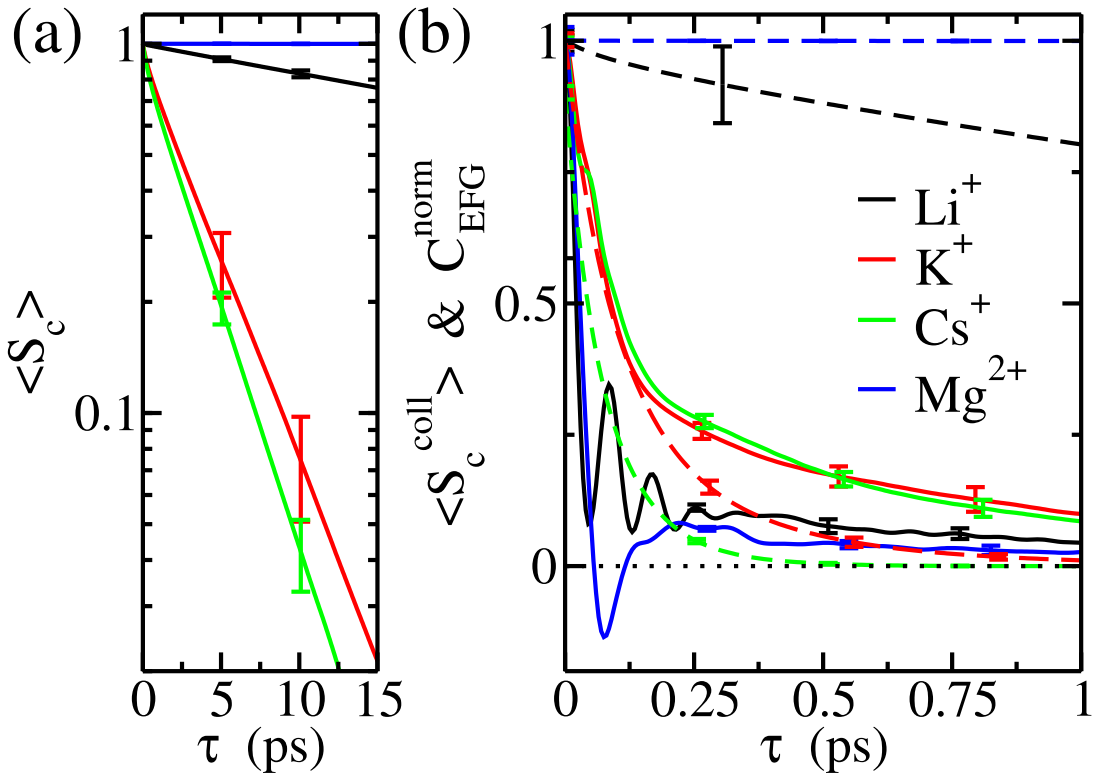

FIG. 3. Survival probability in the first solvation shell: (a) $\left\langle S_{c}\right\rangle$ monitoring entrance/exit events for a given molecule and (b) $\left\langle S_{c}^{c o l l}\right\rangle$ monitoring the first entrance/exit event (dashed lines). In (b) we also report the normalized EFGACF (solid lines). the column of the periodic table (see Table III), reflecting a tighter bound shell with smaller ions due to the stronger electric field. For $\mathrm{Mg}^{2+}$ no exchange events are observed over tens of ps. The survival probability decays much more slowly than the EFG-ACF, so that we can investigate the role of the dynamics of a given molecule within the first solvation shell (see Section III C).

We now consider events of entrance into and exit from the solvation shell from the collective point of view. To that end, we introduce the survival probability of the solvation shell as whole as $\left\langle S_{c}^{\text {coll }}(\tau)\right\rangle$, where $S_{c}^{\text {coll }}(\tau)$ is equal to 1 if the identity of the water molecules in the solvation shell remained unchanged between 0 and $\tau$ and to 0 otherwise. In contrast to $\left\langle S_{c}(\tau)\right\rangle$, which monitors the presence of a given molecule, $\left\langle S_{c}^{\text {coll }}(\tau)\right\rangle$ detects the first entrance or exit event leading to a change in the solvation shell. Figure 3(b) shows the results for $\mathrm{Li}^{+}, \mathrm{K}^{+}, \mathrm{Cs}^{+}$, and $\mathrm{Mg}^{2+}$, together with the EFG-ACF. The situation varies substantially between the ions. The survival probability of the solvation shell decays much more slowly than the EFG-ACF for $\mathrm{Li}^{+}$and $\mathrm{Mg}^{2+}$, over a similar time scale (in particular for the first decay) for $\mathrm{K}^{+}$, and faster for $\mathrm{Cs}^{+}$. This indicates that the decay of the EFG is not due to such entrance or exit events in the cases of $\mathrm{Li}^{+}$and $\mathrm{Mg}^{2+}$, while for $\mathrm{Cs}^{+}$the first entrance or exit occurs faster than the

TABLE III. Characteristic times for the decay of the survival probability $\left\langle S_{c}\right\rangle$ and the rotation $\left(C_{r o t}\right)$ and reorientation $\left(C_{\text {reor }}\right)$ of water molecules. For the latter two, $\tau_{\text {rot }}$ and $\tau_{\text {reor }}$ are determined by an exponential fit between $500 \mathrm{fs}$ and $5 \mathrm{ps}$.

\begin{tabular}{lccc}
\hline \hline Ions & $\tau_{\text {surv }}(\mathrm{ps})$ & $\tau_{\text {rot }}(\mathrm{ps})$ & $\tau_{\text {reor }}(\mathrm{ps})$ \\
\hline $\mathrm{Li}^{+}$ & 54.0 & 26.7 & 13.8 \\
$\mathrm{Na}^{+}$ & 10.5 & 27.3 & 10.8 \\
$\mathrm{~K}^{+}$ & 3.2 & 17.2 & 9.0 \\
$\mathrm{Rb}^{+}$ & 3.1 & 14.7 & 7.7 \\
$\mathrm{Cs}^{+}$ & 2.5 & 14.7 & 7.3 \\
\hline $\mathrm{Mg}^{2+}$ & $\cdots$ & 89.5 & 30.0 \\
\hline \hline
\end{tabular}

decorrelation of the EFG. In addition, the survival probability decays faster as the ion size increases, i.e., following the trend opposite to the EFG. These observations allow us to conclude that the decorrelation of the EFG is not driven by entrance or exit events (which could, in turn, be associated with the decorrelation of the relative orientations). The similar time scales for $\mathrm{K}^{+}$may therefore be fortuitous.

\section{Water dynamics within the first solvation shell}

\section{Rotation and reorientation}

We finally examine the role of the dynamics of water molecules within the solvation shell. The contribution of an individual water molecule to the EFG experienced by the ion depends on the relative position of the molecule with respect to the ion and on its orientation. Noting $\mathbf{r}$ the position of an oxygen of molecule in the frame of the ion, the rotation of the molecule around the ion can be monitored by computing

$$
C_{r o t}(\tau)=\frac{\left\langle\mathbf{u}(\tau) \cdot \mathbf{u}(0) S_{c}(\tau)\right\rangle}{\left\langle S_{c}(\tau)\right\rangle},
$$

where $\mathbf{u}=\frac{\mathbf{r}}{\|\mathbf{r}\|}$ is a unit vector. One can also define a similar correlation function for the reorientation of the molecule by introducing

$$
C_{\text {reor }}(\tau)=\frac{\left\langle\mathbf{v}(\tau) \cdot \mathbf{v}(0) S_{c}(\tau)\right\rangle}{\left\langle S_{c}(\tau)\right\rangle},
$$

where $\mathbf{v}$ is a unit vector along the symmetry axis of the molecule.

Both correlation functions are given in Figure 4(a) for $\mathrm{Li}^{+}$, $\mathrm{K}^{+}, \mathrm{Cs}^{+}$, and $\mathrm{Mg}^{2+}$. The rotation of the water molecule around the ion is slower than the decorrelation of its dipole orientation. Nevertheless, the time scales are comparable for each ion and the decay of both correlation functions extends over tens of picoseconds. This decay is approximately exponential in the 500 fs to 5 ps range, allowing to define the characteristic times $\tau_{\text {rot }}$ and $\tau_{\text {reor }}$ indicated in Table III. The comparable time scales for both processes (with a factor $\sim 2$ for each ion) suggest that the reorientation of the water dipole is correlated with the 

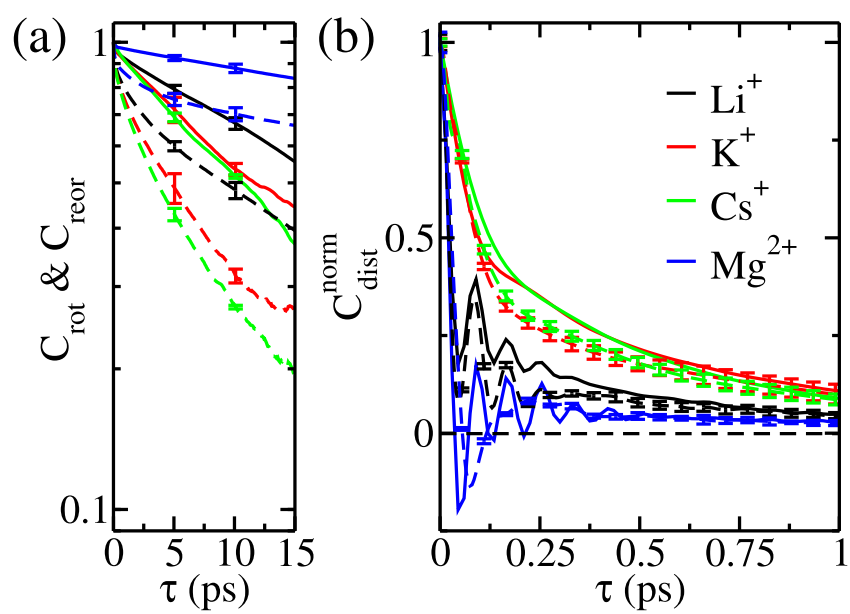

FIG. 4. Normalized correlation functions for (a) the rotation $\left(C_{r o t}\right.$, solid lines) and the self-reorientation $\left(C_{\text {reor }}\right.$, dashed lines) of molecules in the first solvation shell and (b) ion-water distance ( $C_{\text {dist }}^{\text {norm }}$, solid lines). In (b) we also report the normalized EFG-ACF (dashed lines).

rotation of the water molecule around the ion, consistently with the picture of radially oriented dipoles. Both times follow the same trend as for the survival probability $\tau_{\text {surv }}$, again reflecting a more rigid solvation shell for the smaller ions due to the stronger electric field. Note however that for $\mathrm{K}^{+}, \mathrm{Rb}^{+}$, and $\mathrm{Cs}^{+}, \tau_{\text {rot }}$ and $\tau_{\text {reor }}$ are longer than $\tau_{\text {surv }}$, so that the orientational correlation functions reflect the behaviour only of the molecules that stay in the solvation shell longer than the average.

Overall, the rotation-reorientation around the ion occurs slowly compared to the decorrelation of the EFG-ACF. This conclusion supports similar results reported by Engström et al. in the first molecular simulation study of the NMR relaxation rate of these aqueous ions. ${ }^{16}$ It further confirms the weak influence of moment of inertia of the water molecule (see Section III A).

\section{Vibration}

The effect of the ion-water distance $d=\|\mathbf{r}\|$ can be monitored by the correlation function

$$
C_{\text {dist }}(\tau)=\frac{\left\langle d(\tau) d(0) S_{c}(\tau)\right\rangle}{\left\langle S_{c}(\tau)\right\rangle},
$$

which decays on the picosecond time scale towards a plateau which differs from the initial value $\left\langle d^{2}\right\rangle$ only by a few percents (not shown). In order to obtain more insight into the fast initial decay and to compare the results for the different ions, it is convenient to introduce the normalized function

$$
C_{\text {dist }}^{\text {norm }}(\tau)=\frac{C_{\text {dist }}(\tau)-C_{\text {dist }}(\infty)}{C_{\text {dist }}(0)-C_{\text {dist }}(\infty)}
$$

The results for $\mathrm{Li}^{+}, \mathrm{K}^{+}, \mathrm{Cs}^{+}$, and $\mathrm{Mg}^{2+}$ are reported in Figure 4. The decay of $C_{\text {dist }}^{\text {norm }}$ is much faster for the rotation and reorientation and bears strong resemblance to that of the EFG-ACF (Figure 1): The time scales are comparable, the results for $\mathrm{K}^{+}$and $\mathrm{Cs}^{+}$are almost identical, the $\mathrm{Li}^{+}$case displays oscillations with a similar frequency, and a negative part is observed at short times for $\mathrm{Mg}^{2+}$.
Together with the above discussion of other processes, these observations suggest that the ion-water vector vibrates in a picosecond time scale around an "average position" that evolves over longer time scales by rotation around the ion and exit from the solvation shell. The features shared by $C_{\text {dist }}^{\text {norm }}$ and the EFG-ACF indicate that the fluctuations of water molecules around their average position could be the cause of the fluctuations of the EFG. Indeed, as already noted in early molecular simulations studies of the EFG experienced by aqueous cations, the symmetry of the solvation shell may lead to cancellations between various molecules. ${ }^{17}$ In this picture, EFG fluctuations emerge from the small displacement of water molecules which break the overall symmetry of the solvation shell. ${ }^{18}$

\section{Symmetry-breaking fluctuations}

In order to analyze such symmetry-breaking fluctuations, it is necessary to investigate collective effects which are not captured by single-molecule based observables. To address this issue, we introduce here a family of collective variables with suitable symmetry properties and a simple microscopic interpretation,

$$
\begin{aligned}
\mathbf{S}_{p}^{(1)} & =\sum_{i \in \mathcal{S}} \frac{1}{r_{i}^{p}} \frac{\mathbf{r}_{i}}{r_{i}}, \\
\mathbf{S}_{p}^{(2)} & =\sum_{i \in \mathcal{S}} \frac{1}{r_{i}^{p}} \frac{r_{i}^{2} \mathbb{I}-3 \mathbf{r}_{i} \otimes \mathbf{r}_{i}}{r_{i}^{2}},
\end{aligned}
$$

where as above $\mathbf{r}_{i}$ the position of the oxygen of molecule $i$ in the frame of the ion and where the sum runs over molecules in the first solvation shell of the ion, which evolve due to the entrance or exit of molecules. The integer $p$ modifies the weight of the molecules according to their distance from the ion. As an example, $\mathbf{S}_{-1}^{(1)}$ corresponds to the position of the center of mass of the first solvation shell, in the ion frame. $\mathbf{S}_{-2}^{(2)}$ is proportional to the inertia tensor of the solvation shell and $\mathbf{S}_{4}^{(2)}$ is proportional to the EFG created by radially oriented point-dipoles located at the position of the oxygen atoms. For its part, $\mathbf{S}_{0}^{(2)}$ only depends on the relative orientations of the ion-water vectors, not on their norm. This purely orientational order parameter is closely related to the expansion of the water density around the ion using spherical harmonics. All these functions reflect the deviations of the water molecules from their (slowly varying) average position within the solvation shell and thus the changes in the corresponding symmetry.

The normalized auto-correlation functions of $\mathbf{S}_{-1}^{(1)}, \mathbf{S}_{0}^{(2)}$, and $\mathbf{S}_{4}^{(2)}$ are presented in Figure 5 for $\mathrm{Li}^{+}, \mathrm{K}^{+}, \mathrm{Cs}^{+}$, and $\mathrm{Mg}^{2+}$. Similar results and conclusions are obtained for $\mathrm{Na}^{+}$and $\mathrm{Rb}^{+}$, as well as for other functions of the family such as $\mathbf{S}_{-2}^{(2)}$. Except for the center of mass $\mathbf{S}_{-1}^{(1)}$ in the case of $\mathrm{Li}^{+}$, which displays no oscillation at short times, and for $\mathbf{S}_{-1}^{(1)}$ and $\mathbf{S}_{0}^{(2)}$ in the case of $\mathrm{Mg}^{2+}$, which display more oscillations than the EFG-ACF, the decay of all these ACFs is very similar to that of the EFG (see Figure 1). In particular, the decay occurs in two steps, with characteristic times close to that for the EFG, and the trends from one ion to another follow that for the EFG. 


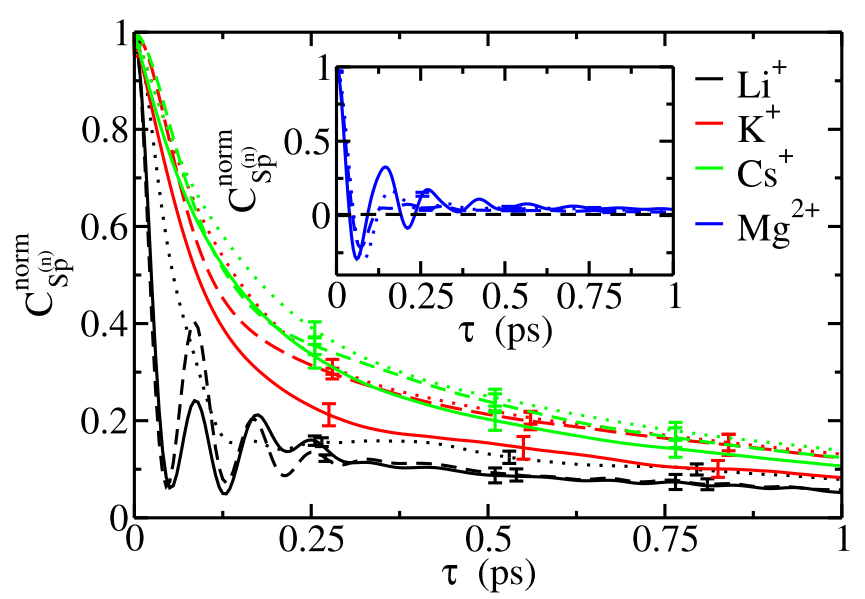

FIG. 5. Normalized correlation functions of different functions of the family defined by Eqs. (9) and (10): $\mathbf{S}_{-1}^{(1)}$ (continuous line), $\mathbf{S}_{0}^{(2)}$ (dotted line), and $\mathbf{S}_{4}^{(2)}$ (dashed line) around different alkali (main figure) and magnesium (inset) cations in water at infinite dilution. Error bars indicate standard deviations over five independent trajectories.

The connection between the EFG and $\mathbf{S}_{4}^{(2)}$ had already been underlined in our previous work introducing the radial dipole model $(\mathrm{RDM}){ }^{23}$ The fact that the other collective variables decay with the same dynamics as the EFG is more surprising. The comparison between the EFG and any of these collective variables reveals that there is no clear correlation between them (not shown), with the notable exception of $\mathbf{S}_{4}^{(2)}$ highlighted in Ref. 23. Nevertheless, the fact that their normalized ACFs look similar reflects that they are all related to the same underlying collective effects-all the more that they all cancel for the same symmetric configurations. In addition, the case of $\mathbf{S}_{0}^{(2)}$ shows that such symmetry-breaking fluctuations are not only due to the radial motion of water. We finally note that the collective variables introduced here may be useful for the development of analytical theories of the dynamics underlying the decay of the EFG-ACF, since they are simpler than the EFG itself.

The importance of the symmetry of the solvation shell for the EFG had been underlined from the static point of view. The present analysis clearly demonstrates this importance also from the dynamic point of view, and as a result for the decay of the EFG-ACF and the NMR relaxation rate. As a final remark, we note that the collective variables $\mathbf{S}_{p}^{(n)}$ are not sufficient to analyze the effect of correlations between water molecules. In the literature, ${ }^{17,18}$ such correlations were analyzed by comparing the self-EFG-ACF (for the EFG due to one of the water molecules) to the overall EFG-ACF. However, the self-EFG-ACF suffers from the same drawbacks as $C_{r o t}(\tau)$ and $C_{d i s t}(\tau)$, with much slower decay than the overall EFG-ACF. ${ }^{17}$ The symmetry of the solvation shell implies a correlation between the slowly varying vibrationally averaged position of the molecules, described in Section III C 2. However, the present analysis does not allow to conclude on the possible correlation between the fast vibration of water molecules around these positions, which are responsible for the decorrelation of the EFG. We leave the analysis of such cross correlations between molecules for further study.

\section{CONCLUSION}

The EFG fluctuations driving the NMR relaxation of quadrupolar cations are mainly due to the dynamics of water molecules in their solvation shell. The dynamics of the ion plays a less important role, with the exception of the short-time dynamics in the lighter $\mathrm{Li}^{+}$and $\mathrm{Mg}^{2+}$ case, for which rattling in the solvent cages results in oscillations of the EFG-ACF. Several microscopic mechanisms that may a priori contribute to the decay of the EFG-ACF occur in fact over too long time scales: entrance/exit of individual water molecules into/from the solvation shell, rotation of a molecule around the ion, or reorientation of the molecule. In contrast, the fluctuations of the ion-water distance are clearly correlated to that of the EFG. Nevertheless, as one may have anticipated, it is not sufficient to consider a single molecule due to the cancellations arising from the symmetry of the solvation shell, and one should account for collective effects.

While the origin of the two-step decay of the EFG$\mathrm{ACF}$ remains to be clarified, the present work underlines the importance of collective symmetry-breaking fluctuations. In that respect, future studies would benefit from using collective variables with appropriate symmetry such as the one introduced here or approaches based on effective normal modes. The latter may in addition provide a connection with $\mathrm{THz}$ spectroscopies which probe the dynamics over comparable time scales and may thus provide further validation of the force field used for MD simulations. Such a combination (effective normal modes and vibrational spectroscopies) might also shed some light on the peculiar behaviour of the $\mathrm{Mg}^{2+}$ cation, which could not be explained here.

Even though contribution to the spectral density at zerofrequency arises from the decay of the EFG-ACF over a few ps for in the present case of infinitely dilute solutions, it should be noted that for finite salt concentrations additional slower mechanisms may also play a role in the NMR relaxation. Interpreting the concentration-dependence of the relaxation rate may require to take into account the relative motion of ions and the possible formation of ion pairs. Similarly, interfaces may also contribute to slower fluctuations of the EFG as the nucleus diffuses toward or away from the surface.

\section{ACKNOWLEDGMENTS}

A.C. acknowledges financial support from UPMC.

\footnotetext{
${ }^{1}$ A. Abragam, The Principles of Nuclear Magnetism (Clarendon Press; Oxford University Press, Oxford [Oxfordshire]; New York, 1983).

${ }^{2} \mathrm{~J}$. Kowalewski, Nuclear Spin Relaxation in Liquids: Theory, Experiments, and Applications, Series in Chemical Physics Vol. 2 (Taylor \& Francis, New York, 2006).

${ }^{3}$ D. Laage, Science 311, 832 (2006).

${ }^{4}$ J. Schmidt, J. Hutter, H.-W. Spiess, and D. Sebastiani, ChemPhysChem 9, 2313 (2008).

${ }^{5}$ V. Calandrini, D. Abergel, and G. R. Kneller, J. Chem. Phys. 133, 145101 (2010).

${ }^{6}$ P. Porion, A. M. Faugère, and A. Delville, J. Phys. Chem. C 112, 11893 (2008).

${ }^{7}$ P. Pyykkö, Mol. Phys. 106, 1965 (2008).

${ }^{8}$ R. Sternheimer, Phys. Rev. 80, 102 (1950).

${ }^{9}$ R. Sternheimer, Phys. Rev. 84, 244 (1951).

${ }^{10}$ A. Carof, M. Salanne, T. Charpentier, and B. Rotenberg, J. Phys. Chem. B 118, 13252 (2014).
} 
${ }^{11}$ H. Hertz, Ber. Bunsen-Ges. Phys. Chem. Chem. Phys. 77, 531 (1973).

${ }^{12}$ J. T. Hynes and P. G. Wolynes, J. Chem. Phys. 75, 395 (1981).

${ }^{13}$ B.-C. Perng and B. M. Ladanyi, J. Chem. Phys. 109, 676 (1998).

${ }^{14}$ S. Engström and B. Jönsson, Mol. Phys. 43, 1235 (1981).

${ }^{15}$ S. Engström and B. Jönsson, J. Magn. Reson. 50, 1 (1982).

${ }^{16}$ S. Engström, B. Jönsson, and R. W. Impey, J. Chem. Phys. 80, 5481 (1984).

${ }^{17}$ J. E. Roberts and J. Schnitker, J. Phys. Chem. 97, 5410 (1993).

${ }^{18}$ M. Odelius and J. Kowalewski, J. Chem. Soc., Faraday Trans. 91, 215 (1995).

${ }^{19}$ R. Baumert, R. Ludwig, and A. Geiger, Mol. Model. Annu. 2, 379 (1996).

${ }^{20}$ S. Badu, L. Truflandier, and J. Autschbach, J. Chem. Theory Comput. 9, 4074 (2013).

${ }^{21}$ K. Aidas, H. Ågren, J. Kongsted, A. Laaksonen, and F. Mocci, Phys. Chem. Chem. Phys. 15, 1621 (2013).

${ }^{22}$ A. Carof, Ph.D. thesis, Université Pierre et Marie Curie, Paris, 2015.

${ }^{23}$ A. Carof, M. Salanne, T. Charpentier, and B. Rotenberg, J. Chem. Phys. 143, 194504 (2015).
${ }^{24}$ S. Tazi, J. J. Molina, B. Rotenberg, P. Turq, R. Vuilleumier, and M. Salanne, J. Chem. Phys. 136, 114507 (2012).

${ }^{25}$ L. X. Dang and T.-M. Chang, J. Chem. Phys. 106, 8149 (1997).

${ }^{26}$ See www.cp2k.org for CP2K developers group.

${ }^{27}$ P. P. Ewald, Ann. Phys. 369, 253 (1921).

${ }^{28}$ J.-P. Ryckaert, G. Ciccotti, and H. J. Berendsen, J. Comput. Phys. 23, 327 (1977).

${ }^{29}$ G. Ciccotti, M. Ferrario, and J.-P. Ryckaert, Mol. Phys. 47, 1253 (1982).

${ }^{30}$ S. Nosé, Mol. Phys. 52, 255 (1984).

${ }^{31}$ S. Nosé, J. Chem. Phys. 81, 511 (1984).

${ }^{32}$ S. Koneshan, R. M. Lynden-Bell, and J. C. Rasaiah, J. Am. Chem. Soc. 120, 12041 (1998).

${ }^{33}$ R. W. Impey, P. A. Madden, and I. R. McDonald, J. Phys. Chem. 87, 5071 (1983).

${ }^{34}$ D. Laage and J. T. Hynes, J. Phys. Chem. B 112, 7697 (2008).

${ }^{35}$ V. Marry, B. Rotenberg, and P. Turq, Phys. Chem. Chem. Phys. 10, 4802 (2008). 